\title{
EDITORIAL
}

\section{Thanks to our reviewers}

\section{Cameron Tropea ${ }^{1}$}

(C) The Author(s) 2021, corrected publication 2021

The Editorial staff of Experiments in Fluids wishes to thank the following reviewers, who have helped to maintain and improve the quality of the journal in 2020.

Christopher Abram
Kareem Ahmed
Jean-Luc Aider
Vladimir Ajaev
Karim Alame
Sergey Alekseenko
Henrik Alfredsson
Stephen Allison
Farrukh S. Alvi
Omid Amili
Seongpil An
Keita Ando
Fabien Anselmet
Nicholas Apazidis
Nasser Ashgriz
Tommaso Astarita
Callum Atkinson
Günter Auernhammer
Franco Auteri
Woutijn Baars
Rudolf Bachert
Christopher Bahr
Honglei Bai
Rio Baidya
Lucia Baker
Lucien Baldas
Arindam Banerjee
Andrew Banko
Joseph Banks
Stephan Bansmer
Philippe Bardet
Robert S. Barlow
Thomas Barois

Christopher Abram

Jean-Luc Aider

Vladimir Ajaev

Sergey Alekseenko

Henrik Alfredsson

Stephen Allison

Omid Amili

Seongpil An

lmet

Tommaso Astarita

Günter Auernhammer

Christopher Bahr

Honglei Bai

Rio Baidya

Lucia Baker

Lucien Baldas

Arindam Banerjee

Andrew Banko

Joseph Banks

Stephan Bansmer

Philippe Bardet

Thomas Barois
Nicholas Apazidis

Callum Atkinson

Diogo Barros
Saptarshi Basu
Andreas Bauer
André Bauknecht
Alexander Bazilevsky
Stefan Becker
Thomas Behrendt
Jesse Belden
Steven J. Beresh
Zachary Berger
Edouard Berrocal
Fulvio Bertocchi
Chunwei Bi
Weitao Bi
Alessandro Bianchini
Philippe Blanc-Benon
Gianluca Blois
Guido Boffetta
Douglas Bohl
Benjamin Böhm
Richard Bomphrey
Daniel Bonn
Jeffrey Bons
Joakim Bood
Daniel Borup
Wouter Bos
Johannes Bosbach
Lydia Bourouiba
Isaac Boxx
Andreas Braeuer
Robert Breidenthal
Christian Breitsamter
Guenter Brenn

Cameron Tropea

tropea@sla.tu-darmstadt.de

1 Darmstadt, Germany

Kenneth Breuer
Matthew Bross
Paul Bruce
Philippe Brunet
Gilles Bruneux
André Brunn
Martin Bruschewski
John Buchner
Yannick Bury
David Buttsworth
Oliver Buxton
Christopher Cadou
Weiwei Cai
Raul Bayoan Cal
Francesco Camana
Roberto Camussi
Gennaro Cardone
Matteo Carpentieri
Douglas Carter
Campbell Carter
Guillaume Castanet
Thomas Castelain
Jose Castrejon-Pita
Ian P Castro
John Cater
André Cavalieri
Steven Ceccio
Oksan Cetiner
John Charonko
George Chase
Fang Chen
Jun Chen
Lin Chen
Valery Chernoray
Vladimir Chirkov
Chang Kyoung Choi
Kwing-So Choi
Christian Cierpka


Ivan Cimrák

Francois Clemens

Noel Clemens

James Coder

Filippo Coletti

Tim Colonius

Chris Combs

Patrick Comiskey

Jonathan Cooper

Laurent Cordier

Thomas Corpetti

Francois Coulouvrat

Edwin Cowen

Fabio Cozzi

Thomas Cubaud

Cathal Cummins

Daniel Cuppoletti

Dana Dabiri

Werner Dahm

Stuart Dalziel

Anton Darhuber

Kyle Daun

Thierry Dauxois

Laurent David

Todd Davidson

James Dawson

Scott Dawson

Antoine Dazin

Roeland de Kat

Prabhath De Silva

Anne De Wit

Mohan D. Deshpande

Francesco Di Natale

Nicolas Dietrich

Georg Dietze

Stefano Discetti

Henda Djeridi

Arthur Dogariu

Con J. Doolan

Thomas Dreier

Philippe Druault

James Duncan

Jared Dunnmon

Vibhav Durgesh

Craig Dutton

Dominik Ebi

Sven Eckert

Daniel Edgington-Mitchell

Brian Elbing

Jeff Eldredge

Veronica Eliasson

Christopher Elkins

Gerrit Elsinga
Casey Fagley

Todd Fansler

Mohamed Farhat

John Farnsworth

Aamir Farooq

Lihao Feng

Lorenzo Ferrari

Carlos Simão Ferreira

Manuel Ferreira

Erhan Firat

Daniele Fiscaletti

Karen Flack

Jan-Bert Flor

John Foss

Jean-Marc Foucaut

Fabrice Foucher

Nicolas Francois

Jonathan Frank

Udo Fritsching

Song Fu

Thomas Fuchs

Koji Fukagata

Matthew Fulghum

Shumpei Funatani

Noriyuki Furuichi

Lian Gan

Ranjan Ganguly

Nan Gao

Qi Gao

Yi Gao

Ricardo García-Mayoral

Anthony Gardner

Steve Garoff

Manolis Gavaises

Pascal Gehlert

Yves Gervais

Sina Ghaemi

Mohammad Ghalambaz

Rogier Giepman

Patrick Gnemmi

Christopher Goldenstein

Guillaume Gomit

Ashok Gopalarathnam

Robert Gordon

Mark Gragston

Michael Graham

Will R Graham

Michela Gramola

Samuel Grauer

Melissa Green

Richard Green

David Greenblatt

Christof Gromke
Guillaume Grossir

Sven Grundmann

Michele Guala

Thibault Guiberti

Amandine Guissart

Melika Gul

Ali Gülhan

Ismet Gursul

Thomas Haeber

Rainer Hain

Wang Han

Ronald Hanson

Michael Hargather

John Harvey

Yassin Hassan

Fatemeh Hassanipour

Stefan Haxter

Kosuke Hayashi

Chuangxin $\mathrm{He}$

Adam Head

Robert Jason Hearst

James Heineck

Martin Heinold

Michael Heisel

Dominique Heitz

Maziar Hemati

Jason Hindmarsh

Avraham Hirschberg

Simone Hochgreb

Michael Hölling

Markus Holzner

Jiarong Hong

Shigeo Hosokawa

Kazunori Hosotani

Peter Huang

Marcus Hultmark

Jeanette Hussong

Nicholas Hutchins

Andrea Ianiro

Joseph Ibrahim

Yuka Iga

Tomonori Ihara

Hitoshi Ishikawa

Ian Jacobi

Bernd Jaehne

Gopal Jagadeesh

Saad Jahangir

Kartik Jain

Sahar Jalal

Girish Jankee

Thierry Jardin

Vincent Jaunet

Anatol Jaworek 
Jan Jedelsky

Mark Jermy

Joseph Jewell

Lin Jiang

Christophe Josserand

Khalid Juhany

Daniel Juvé

Sebastian Kaiser

Masaharu KamedaA

Alexandra Kameke

Akiko Kaneko

Tatsuya Kawaguchi

Yasuo Kawaguchi

Yutaka Kazoe

Douglas Kelley

Franck Kerherve

Felix Kern

Kevin Kevin

Iltai Kim

Hyoungsoo Kim

Kyung Chun Kim

Mark Kimber

Jonas Kind

Rudibert King

Branden Kirchner

Tania Kirmse

Johannes Kissing

Atsuhide Kitagawa

Daphne Klotsa

Jeff Knowles

Kevin Knowles

Yoshimitsu Kobashi

Rainer Koch

Shunsuke Koike

Jörg König

Piotr Korczyk

Charlotte Kotas

Marios Kotsonis

Tomas Kowalewski

Melissa Kozul

Elias Kristensson

Anjaneyulu Krothapalli

Paul Krueger

Dominik Krug

Simon Kuhn

Ichiro Kumagai

Rajan Kumar

Melike Kurt

Chris Lai

Gracia Lamanna

Angeliki Laskari

Timothy Lau

Stuart Laurence
Philippe Lavoie

Nicholas Lawson

Trung Le

Tanguy Le Borgne

Bertrand Lecordier

Minwook Lee

Sangseung Lee

Sang Joon Lee

Tim Lee

Philippe Leick

Fabrice Lemoine

David-Elie Levy

Zhongshan Li

Alex Liberzon

Anke Lindner

Mark Linne

Mario Lino da Silva

Jesse Little

Hai-Feng Liu

Tianshu Liu

Xiaofeng Liu

Marco Lorenzini

Kevin Lowe

Jens-Michael Löwe

James Luckring

Mitul Luhar

Staffan Lundström

Zhenbing Luo

Gaetano Magnotti

Simo Makiharju

Michael Manhart

Keefe B. Manning

Raffaello Mariani

Christos Markides

Dmitriy Markovich

Robert Martinuzzi

Romain Mathis

Manikandan Mathur

Nicolas Mazellier

Mark McCorquodale

Jacob McFarland

Matthew McGilvray

Tim McIntyre

Beverley McKeon

Albert Medina

Wolfgang Meier

Ricardo Mejia-Alvarez

Latunia Melton

Etienne Memin

Viren Menezes

Jan Menser

Marie-Claire Merienne

Knud Erik Meyer
Terrence Meyer

Jason Meyers

Aliaksndr Mialdun

Jiun-Jih Miau

Mark Miller

Massimo Miozzi

Shuichiro Miwa

Hideaki Monji

Jose Montanero

Hideo Mori

Philip Morris

Scott C. Morris

Jonathan Morrison

Chris Morton

Karen Mulleners

Hideki Murakawa

Shigeru Murata

Stefano Musacchio

Hiroki Nagai

Yoshitsugu Naka

Vinod Narayanan

Venkat Narayanaswamy

Vivek Narsimhan

Christian Nayeri

Andrew Neely

Tze How New

Claudia Nicolai

Francois Nicolas

Takasi Nisisako

Bernd Noack

Holger Nobach

David Nobes

Taku Nonomura

Caroline Nore

Venkata Nori

Matteo Novara

Martin Obligado

Dominik Obrist

Philippe Odier

Kazuo Ohmi

Yoshihiko Oishi

Kohei Okita

Simon Olivier

Michael Olsen

Ramis Örlü

Alfred R Osborne

Michael Oschwald

Nicholas Ouellette

Austin Overmeyer

Yuta Ozawa

Zhao Pan

Tianyu Pan

Roberto Paoli 
Dimitrios Papavassiliou

Hyun Jin Park

Hyungmin Park

Jinsoo Park

Nick Parziale

Mathias Paschen

Martin Passmore

Luc Pastur

Raul Payri

Joachim Peinke

Peter Pelz

Di Peng

Mustafa Percin

Francisco Pereira

Brian Peterson

Jimmy Phillip

Filippo Pierini

Fernando Pinho

Sébastien Piponniau

Markus Piro

Robert W. Pitz

Marco Placidi

Bérengère Podvin

Christian Poelma

Gerard Poitras

Bernard Pouligny

Sotiris Pratsinis

Katherine Prestridge

Nimish Pujara

Ishwar Puri

Hui-He Qiu

Mark Quinn

Willie Quinn

Rolf Radespiel

Sina Rafati

Markus Raffel

Vrishank Raghav

Daniele Ragni

Lalit Rajendran

Manikandan Ramasamy

Robin Ras

Manuel Reddemann

Yukun Ren

Antoine Renaud

Mark Rennie

David Reuss

Gal Ribak

Mattias Richter

Gregory Rieker

Georgios Rigas

Akira Rinoshika

Leif Ristroph

David Rival
Donald Rockwell

Thomas Roesgen

Veronique Roig

Ilia Roisman

Massimiliano Rossi

David Rothamer

Jonathan Rothstein

Gem Rotte

Gauthier Rousseau

Alexei Rozhkov

Kshitij Sabnis

John Sader

Rakesh Sahu

Jun Sakakibara

Milad Samie

Toshiyuki Sanada

Tobias Sander

Abhilash Sankaran

Akihiro Sasoh

Thomas Sattelmayer

Alexey Saveliev

Fulvio Scarano

Walter Schäfer

Daniel Schanz

Sven Scharnowski

Martin Schiemann

Daniel Schiepel

Michael Schlueter

Peter Schmid

Bryan Schmidt

Steven Schneider

Jan Schramm

Ferry Schrijer

Andreas Schröder

Erich Schülein

William Schultz

Jörg Schumacher

Andrea Sciacchitano

David Sedarsky

Andy Sederman

Antonio Segalini

Ashis Sen

Thomas Seon

Soumyadip Sett

Gary Settles

Mark Sheplak

John Shrimpton

Volker Sick

Claus Sieverding

Pieter Sijtsma

Suman Sinha-Ray

Sumit Sinha-Ray

Magnus Sjöberg
Barton Smith

Alexander Smits

Craig Snoeyink

Hossein Sojoudi

Julio Soria

Geoffrey Spedding

Adrian Spencer

Atul Srivastava

Ralf Stark

Victor Starov

Christopher Staszel

Lester Su

Kazuhiko Suga

Kazuyasu Sugiyama

David Sumner

Zhengzhong Sun

Rainer Suntz

Jeffrey Alan Sutton

Kosuke Suzuki

Josue Sznitman

Ryszard Szwaba

Mark Tachie

Yoshiyuki Tagawa

Hiroyuki Takahira

Masahiro Takei

Chao Tan

Hideyuki Tanno

Sedat Tardu

Yuji Tasaka

Manilo Tassieri

Keith Taylor

Alex Taylor

Yi Tee

Vaclav Tesar

Lionel Thomas

Marie-Jean Thoraval

Sigurdur Thoroddsen

James Threadgill

Brian Thurow

Charles Tinney

Takuya Tsuji

Eleonora Tubaldi

Ankit Tyagi

Lawrence Ukeiley

Thomas Underwood

Hooman Vahedi Tafreshi

Vincent Valeau

Andres Valencia

Bas van Oudheusden

Leon Vanstone

Evan Variano

Chistos Vassilicos

Subith Vasu 
Clara Velte

Lakshmi Venkatakrishnan

Shashi Verma

Siddhartha Verma

Michel Versluis

Ilia Vichev

Francesco Viola

Ignazio Maria Viola

Preetinder Virk

Pavlos Vlachos

Romain Volk

Greg Voth

Evgeny Votyakov

Claus Wagner

Paulo Waltrich

An-Bang Wang

Cong Wang

Zhijin Wang

Tomoaki Watamura

Masao Watanabe

Satoshi Watanabe
Neal Watkins

Donald Webster

Nathaniel Wei

Daniel Weiss

Julien Weiss

Peter Wellens

Wenguo Weng

Michael Wensing

Mark Wernet

Jeanette Wheeler

Martin White

Bernhard Wieneke

David Williams

Rhys Williams

Claus Christian Wolf

Thomas Wondrak

Nicholas Worth

Rene Woszidlo

Huixuan Wu

Xuecheng Wu

Werner Wuerz
Keqing Xia

Chao Xu

Jie Xu

Xiangchun Xuan

Gouji Yamada

Serhiy Yarusevych

Sam Sukgoo Yoon

Anna Young

Hossein Zare-Behtash

Wenshuo Zhang

Yang Zhang

Lihao Zhao

Benzhong Zhao

Bo Zhou

Tongming Zhou

Wu Zhou

Lars Zigan

Oleg Zikanov

Gennady Ziskind

Funding Open Access funding enabled and organized by Projekt DEAL.

Open Access This article is licensed under a Creative Commons Attribution 4.0 International License, which permits use, sharing, adaptation, distribution and reproduction in any medium or format, as long as you give appropriate credit to the original author(s) and the source, provide a link to the Creative Commons licence, and indicate if changes were made. The images or other third party material in this article are included in the article's Creative Commons licence, unless indicated otherwise in a credit line to the material. If material is not included in the article's Creative Commons licence and your intended use is not permitted by statutory regulation or exceeds the permitted use, you will need to obtain permission directly from the copyright holder. To view a copy of this licence, visit http://creativecommons.org/licenses/by/4.0/.

Publisher's Note Springer Nature remains neutral with regard to jurisdictional claims in published maps and institutional affiliations. 